EPJ Web of Conferences 59, 06004 (2013)

DOI: $10.1051 /$ epjconf/20135906004

(C) Owned by the authors, published by EDP Sciences, 2013

\title{
Al plasma jet formation via ion stream compression by surrounding low-Z plasma envelope
}

\author{
T. Pisarczyk ${ }^{1}$, A. Kasperczuk ${ }^{1}$, T. Chodukowski ${ }^{1}$, Z. Kalinowska ${ }^{1}$, \\ S.Yu. Guskov², N.N. Demchenko², O. Renner ${ }^{3}$, E. Krousky ${ }^{3}$, M. Pfeifer ${ }^{3}$, \\ K. Rohlena ${ }^{3}$, J. Skala ${ }^{3}$, J. Ullschmied ${ }^{4}$, D. Klir ${ }^{5}$, J. Kravarik ${ }^{5}$, P. Kubes ${ }^{5}$, \\ K. Rezac ${ }^{5}$ and P. Pisarczyk ${ }^{6}$
}

1 Institute of Plasma Physics and Laser Microfusion, 23 Hery St., 00-908 Warsaw, Poland

2 P.N. Lebedev Physical Institute of RAS, 53 Leninsky Ave., 119991 Moscow, Russia

3 Institute of Physics ASCR, v.v.i., Na Slovance 2, 18221 Prague 8, Czech Republic

4 Institute of Plasma Physics ASCR, v.v.i., Za Slovankou 3, 18200 Prague 8, Czech Republic

${ }^{5}$ Czech Technical University in Prague, FEE, Prague, Czech Republic

${ }^{6}$ Warsaw University of Technology, ICS, 15/19 Nowowiejska St., 00-665 Warsaw, Poland

\begin{abstract}
In our earlier papers it was demonstrated that the plasma pressure decreases with the growing atomic number of the target material. In this context a question arose about the possibility to collimate the $\mathrm{Al}$ plasma outflow by using the plastic plasma as a compressor. For that purpose a plastic target with an Al cylindrical insert of $400 \mu \mathrm{m}$ in diameter was used. The experiment was carried out at the PALS laser facility. The laser provided a $250 \mathrm{ps}$ (FWHM) pulse with the energy of $130 \mathrm{~J}$ at the third harmonic frequency $\left(\lambda_{3}=0.438 \mu \mathrm{m}\right)$. The focal spot diameters $\left(\Phi_{\mathrm{L}}\right) 800,1000$, and $1200 \mu \mathrm{m}$ ensured predominance of the plastic plasma, its transversal extension being large enough for the effective Al plasma compression. To study the $\mathrm{Al}$ plasma stream propagation and its interaction with the plastic plasma, a 3-frame interferometric system and 4-frame $\mathrm{x}$-ray camera were used. The information on distribution of electron temperature in the outflowing Al plasma was provided by x-ray spectroscopy. The experimental results reported in the paper are discussed by virtue of a simple theoretical analysis.
\end{abstract}

\section{INTRODUCTION}

In 2006 we reported a simple method of plasma jet generation based on irradiation of flat massive targets with atomic number $\mathrm{Z} \geq 29$ ( $\mathrm{Z}=29$ corresponds to $\mathrm{Cu}$ ) by the third harmonic of a single partly defocused laser beam [1]. Our experiments at the Prague Asterix Laser System (PALS) laser facility have proved that annular target irradiation plays a decisive role in the plasma jet forming [2]. However, this mechanism acts properly only in the case of heavy target materials. If the target is made of light materials like plastic $(\mathrm{CH})$ or $\mathrm{Al}$, no plasma jets are observed, despite the initial laser intensity distribution is the same. However, our investigations of the plasma stream emitted from a joint of light and heavy target materials ( $\mathrm{Al}-\mathrm{Cu}$ or $\mathrm{CH}-\mathrm{Cu}$ ) [3] have shown that the plasma jet is not propagating normally to the target surface, but it is deflected to the side of the heavier material. The theoretical analysis [?] allowed us to deduce that the ratio of plastic and copper plasma pressures amounts to 1.35 and to conclude that the lighter is the plasma the higher is its pressure. Simultaneously a natural question arose about the possibility of the Al plasma jet creation by using plastic plasma as a compressor. The compression of ablation plasma by the plastic plasma envelope opens possibility for exploiting the plasma jets of low atomic number material e.g. in laboratory astrophysics.

This is an Open Access article distributed under the terms of the Creative Commons Attribution License 2.0, which permits unrestricted use, distribution, and reproduction in any medium, provided the original work is properly cited. 

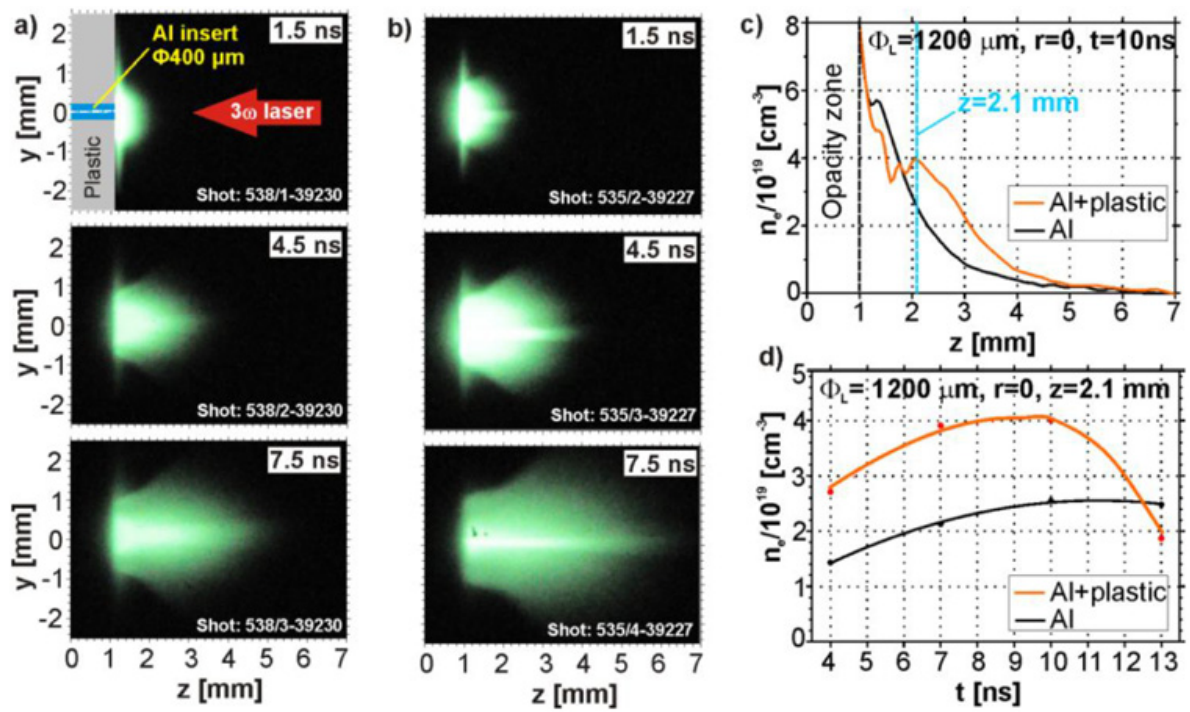

Figure 1. Sequences of $\mathrm{X}$-ray plasma images formed at $\mathrm{Al}$ (a) and plastic target with $\mathrm{Al}$ cylindrical insert (b) and diagrams characterizing the electron density distribution along the axis for the $\mathrm{Al}$ target and the plastic target with the $\mathrm{Al}$ insert (c), and the density evolution (d) with time.

\section{EXPERIMENTAL SETUP, CONDITIONS AND RESULTS}

The experiment was carried out with the use of the PALS iodine laser facility. The plasma was generated by a single beam of the third harmonic laser radiation $(\lambda=0.438 \mu \mathrm{m})$ with parameters: $\mathrm{E}_{\mathrm{L}}=130 \mathrm{~J}, \tau \approx$ $250 \mathrm{ps}$ and focal spot diameters $\Phi_{\mathrm{L}}=800,1000$, and $1200 \mu \mathrm{m}$. The laser irradiated a plastic target with an $\mathrm{Al}$ cylindrical insert of $400 \mu \mathrm{m}$ in diameter.

Two diagnostics have been used to study the Al plasma jet formation: (i) a three-frame interferometric system using the second laser harmonic $(\lambda=0.638 \mu \mathrm{m})$ and a four-frame $\mathrm{x}$-ray pinhole camera, registering the soft $\mathrm{x}$-ray plasma radiation in the range of $10-1000 \mathrm{eV}$. The exposure time of the $\mathrm{x}$-ray camera was below $2 \mathrm{~ns}$. The electron temperature distribution in the outflowing Al plasma was provided by $\mathrm{x}$-ray spectroscopy.

To ensure that the plastic plasma volume is large enough for effective Al plasma compression, we started our investigations with $\Phi_{\mathrm{L}}=800 \mu \mathrm{m}$. Then the focal spot diameter was gradually increased by $200 \mu \mathrm{m}$ up to $1200 \mu \mathrm{m}$. The interferometric measurements have shown that the Al plasma compression grows with increasing the focal spot diameter, the best result corresponding to the largest $\Phi_{\mathrm{L}}$. Therefore our presentation and discussion concentrates on results obtained for $\Phi_{\mathrm{L}}=1200 \mu \mathrm{m}$.

In order to distinguish the Al plasma from the whole plasma bulk probed by the interferometry, an $\mathrm{x}$-ray framing camera was used. This diagnostic is capable of resolving the $\mathrm{Al}$ and plastic plasma components due to a large difference in their radiation intensities, as demonstrated by sample results presented in Fig.1a,b. The Al plasma jet in a form of a narrow bright streak can clearly be distinguished on the plastic plasma background, Fig. 1b. In the early period of the plasma evolution, the constrained Al plasma jet forms close to the target surface. Its diameter is approximately equal to $100 \mu \mathrm{m}$ and it propagates with an average velocity of $7 \times 10^{7} \mathrm{~cm} / \mathrm{s}$. This velocity is considerably larger than the axial velocity of the pure Al plasma $\left(\sim 5 \times 10^{7} \mathrm{~cm} / \mathrm{s}\right)$. It means that the interaction of the plastic and $\mathrm{Al}$ plasmas results not only in the Al plasma jet creation but also in its acceleration.

Differences between the plasma configurations of the pure $\mathrm{Al}$ plasma and the $\mathrm{Al} /$ plastic plasma have been determined from the interferometric measurements. In Fig. 1c, the diagrams of electron density distribution along the axis for the bare $\mathrm{Al}$ target and the cylindrical $\mathrm{Al}$ target embedded into plastic are 



Figure 2. X-ray spectroscopic results: a) spatially resolved K-shell spectral emission, and b) the electron temperature corresponding to both target types.

drawn. For $\mathrm{z}>2 \mathrm{~mm}$, the $\mathrm{Al}$ plasma compression leads to an increase in the on-axis electron density by approximately a factor of 2 in comparison with that of the plasma launched on the bare Al target. On the other hand, the life-time of such a plasma configuration is relatively short and the electron density decreases at the axis. Changes of the electron density at the axis for both targets used vs. time corresponding to $\mathrm{z}=2.1 \mathrm{~mm}$, i.e., at the electron density local maximum (see Fig. 1c), are presented in Fig. 1d. The Al plasma compression at this cross-section lasts about $10 \mathrm{~ns}$. Later on, the electron density drops considerably below the value characteristic for the bare Al plasma. In contrast to this, the latter plasma conserves its structure for longer time.

The information on distribution of electron temperature in the outflowing Al plasma was provided by x-ray spectroscopy [4]. The Al K-shell self-emission spectra were recorded using the imaging-mode $\mathrm{x}$-ray spectrometer equipped with a mica (004) crystal spherically bent to a radius of $150 \mathrm{~mm}$. The calibrated x-ray spectra corresponding to the above described laser-irradiated Al targets are presented in Fig. 2a. The intensity ratios of selected spectral transitions in the $\mathrm{H}$ - and He-like ions provide a wellestablished method for rough estimates of the electron temperature $\mathrm{T}_{\mathrm{e}}$ [5]. Close to the target surface, the best fit $\mathrm{T}_{\mathrm{e}}$ values were determined from different combinations of parent lines and their satellites, at larger distances the ratios of dominant lines were used. The electron temperatures determined from spatially resolved spectral lineouts of bare and constrained-flow Al targets are shown in Fig. $2 \mathrm{~b}$. The results of electron temperature measurement can be explained as follows: The lower electron temperature of $\mathrm{Al}$ plasma at the target surface in the case of the constrained-flow Al target results from the lower laser radiation intensity in the center. As mentioned above, the laser intensity distribution in the transverse beam cross-section has a depression, which just covered the Al insert. The higher electron temperature in the case of the bare $\mathrm{Al}$ target corresponds to the higher intensity of the laser radiation in the off-centre region. With growing distance from the target surface the electron temperature of the $\mathrm{Al}$ plasma launched on the constrained-flow Al target becomes higher than that produced from the pure $\mathrm{Al}$ target. The predominance of this temperature over the other is induced by the Al plasma compression, which grows with time. It seems that this predominance should be considerably higher for $\mathrm{z}>2 \mathrm{~mm}$, where the Al plasma compression is very effective. However the resonance lines intensities were too low for the reliable electron temperature determination at these distances. Nevertheless, the tendency of branching off the electron temperatures with the increasing distance from the target is clearly seen.

\section{THEORETICAL ANALYSIS OF THE EXPERIMENTAL RESULTS}

According to results presented in the paper [6], the ratio of evaporated masses of aluminium (Al) and plastic $(\mathrm{CH})$, i.e. mass per irradiated surface unit in $\mathrm{g} / \mathrm{cm}^{2}$, can be estimated by formula:

$$
\frac{\Delta m_{A l}}{\Delta m_{C H}}=\left(\frac{\chi_{A l}}{\chi_{C H}}\right)^{1 / 2},
$$


where $\chi=\kappa \rho / C_{V}$ is a coefficient of thermal conductivity, $\rho$ is the initial target density, $C_{V}$ is the specific heat, and $\kappa$ is the Spitzer's coefficient of electron heat conductivity. The estimation gives $\Delta m_{A l} / \Delta m_{C H}=1.99$. At later time the energy absorbed by the plasma is completely transformed into the kinetic energy of the plasma expansion, the estimation of the ratio of the plastic and the $\mathrm{Al}$ plasma expansion velocities gives the value $\left.\mathrm{u}_{\mathrm{CH}}^{*} / \mathrm{u}_{\mathrm{Al}}^{*}=\Delta m_{A l} / \Delta m_{C H}\right)^{1 / 2}=1.41$. Since the plastic plasma overtakes the $\mathrm{Al}$ one, it induces an enhancement of the plastic plasma pressure beyond that of the $\mathrm{Al}$ plasma. It results in the plasma motion towards the axis. The time-averaged plasma pressure at the axis in the case of the radial compression can be estimated as $\left(p_{1}+p_{0}\right) / 2$, where $p_{1}=p_{0}\left(\rho_{1} / \rho_{0}\right)^{\gamma}$. Due to the low plasma temperature the thermal conductivity is neglected. Difference in the plasma pressures $\left(p_{1}+p_{0}\right) / 2-p_{0}=\left(p_{1}-p_{0}\right) / 2$ leads to the plasma reflection from the axis. Consequently, the equation for the plasma momentum can be written as follows:

$$
2 u_{r} \Delta m_{r A l}=\frac{p_{0}}{2}\left[\left(\frac{\rho_{1}}{\rho_{0}}\right)^{\gamma}-1\right] \Delta t,
$$

where: $\Delta t=R_{A l} / c_{s}, c_{S}=8.43 \times 10^{6} \mathrm{~cm} / \mathrm{s}-$ sound speed. The equation (2) gives the compression value $\rho_{1} / \rho_{0}=1.7$, which is close to the experimental value $n_{e 1} / n_{e 0}=1.6$ observed at $10 \mathrm{~ns}$ (see Fig. 3). When the process of the plasma focusing is completed, the plasma reflection from the axis occurs and the plasma radial velocity changes its sign.

\section{CONCLUSIONS}

In this work we have demonstrated the possibility of the $\mathrm{Al}$ plasma jet creation by using the plastic plasma as a compressor. In our experiments we took an advantage from the fact that the lighter is the plasma, the higher is its pressure. Based on theoretical analysis, we can conclude that the pressure difference in plasmas with different atomic numbers results from differences in their expansion features. The estimation of the ratio of the plastic and $\mathrm{Al}$ plasma expansion velocities gives the value of 1.41. As a result, the plastic plasma overtakes the $\mathrm{Al}$ one. It induces an enhancement of the plastic plasma pressure beyond the pressure of $\mathrm{Al}$ plasma and, in consequence, the Al plasma motion towards the axis.

The authors gratefully acknowledge the support received from the EC 7th FP under Grant Agreement $\mathrm{N}^{\circ} 228334$ (Laserlab-Europe), Czech MSMT under the grants $\mathrm{N}^{\circ}$ LC528 and 7E09092, Czech Science Foundation Grant No. P205/10/0814, and the Ministry of Science and Higher Education MNiSZW, Poland, under Grant No N N202 130639.

\section{References}

[1] A. Kasperczuk, T. Pisarczyk, S. Borodziuk, J. Ullschmied, E. Krousky, K. Masek, K. Rohlena, J. Skala and H. Hora, Phys. Plasmas 13, 062704-1 (2006)

[2] A. Kasperczuk, T. Pisarczyk, M. Kalal, J. Ullschmied, E. Krousky, K. Masek, M. Pfeifer, K. Rohlena, J. Skala and P. Pisarczyk, Appl. Phys. Letter 94, 081501 (2009)

[3] T. Pisarczyk, A. Kasperczuk, M. Kalal, S.Yu. Gus'kov, J. Ullschmied, E. Krousky, K. Masek, M. Pfeifer, K. Rohlena, J. Skala, and P. Pisarczyk, Proceedings of $35^{\text {th }}$ EPS Conference on Plasma Phys., Hersonissos, 9-13 June 2008, ECA 32, P- 1.118 (2008)

[4] A. Kasperczuk, T. Pisarczyk, J. Badziak, S. Borodziuk, T. Chodukowski, S.Yu. Gus'kov, N.N. Demchenko, J. Ullschmied, E. Krousky, K. Masek, M. Pfeifer, K. Rohlena, J. Skala, and P. Pisarczyk, Phys. Plasmas 17, 114505-1 (2010) 


\section{IFSA 2011}

[5] O. Renner, I. Uschmann, and E. Förster, Laser Part. Beams, 22, 25-28 (2004)

[6] H.-K. Chung, M.H. Chen, W.L. Morgan, Y. Ralchenko, and R.W. Lee, High Energy Density Physics 1, 3-12 (2005)

[7] A. Kasperczuk, T. Pisarczyk, N.N. Demchenko, S.Yu. Gus'kov, M. Kalal, J. Ullschmied, E. Krousky, K. Masek, M. Pfeifer, K. Rohlena, J. Skala, and P. Pisarczyk, LPB 27, 415 (2009) 